\title{
Electroacupuncture at the governor vessel and bladder meridian acupoints improves postmenopausal osteoporosis through osteoprotegerin/RANKL/RANK and Wnt/ $\beta$-catenin signaling pathways
}

\author{
XUEFENG ZHENG ${ }^{1}$, GUANGWEN WU ${ }^{1}$, YAN NIE $^{2}$ and YANPING LIN ${ }^{1}$ \\ ${ }^{1}$ Acupuncture College, Fujian University of Traditional Chinese Medicine, Fuzhou, Fujian 350122; \\ ${ }^{2}$ The Affiliated Traditional Chinese Medicine Hospital of Fuzhou, Fujian University \\ of Traditional Chinese Medicine, Fuzhou, Fujian 350001, P.R. China
}

Received December 25, 2014; Accepted May 15, 2015

DOI: $10.3892 /$ etm.2015.2553

\begin{abstract}
Previous studies have demonstrated that pretreatment with electroacupuncture (EA) at the zusanli/ST36 and sanyinjiao/SP6 acupoints prevents ovariectomy-induced osteoporosis in rats; however, the therapeutic effects of EA at the governor vessel (GV) and bladder meridian (BL) acupoints remain unclear. In the present study, the effects of EA at the GV4, GV6, BL20 and BL23 acupoints on the bones of ovariectomized (OVX) rats were investigated to identify the pathways that mediate the action of EA on the bones. A postmenopausal osteoporosis model was established by performing an ovariectomy in six-month-old female Sprague Dawley rats. Following the ovariectomy, EA treatment was administered once per day for 90 days, with an interval of 5 days per 10 days. The changes in the serum levels of estradiol (E2) and the bone turnover markers, osteocalcin (OC) and tartrate-resistant acid phosphatase 5 b (TRACP 5b), were determined, while the bone mineral density (BMD) of the lumbar vertebra and the histomorphology of the femur were observed. Furthermore,
\end{abstract}

Correspondence to: Professor Yanping Lin, Acupuncture College, Fujian University of Traditional Chinese Medicine, 1 Qiuyang Road, Fuzhou, Fujian 350122, P.R. China

E-mail: fjlyp2014@126.com

Abbreviations: GV, governor vessel; BL, bladder meridian; PMOP, postmenopausal osteoporosis; OVX, ovariectomy; EA, electroacupuncture; BMD, bone mineral density; OC, osteocalcin; TRACP 5b, tartrate-resistant acid phosphatase 5b; OPG, osteoprotegerin; RANKL, receptor activator of nuclear factor- $\kappa \mathrm{B}$ ligand; E2, estradiol; LRP, low-density lipoprotein receptor-related protein; Runx, runt-related transcription factor; HRT, hormone replacement therapy; $\mathrm{H} \& \mathrm{E}$, hematoxylin and eosin

Key words: postmenopausal osteoporosis, ovariectomized rat, electroacupuncture, osteoprotegerin, receptor activator of nuclear factor- $\kappa \mathrm{B}$ ligand, Wnt, $\beta$-catenin the expression of osteoprotegerin (OPG) and receptor activator of nuclear factor- $\mathrm{\kappa B}$ ligand (RANKL), which are involved in the OPG/RANKL pathway, were detected by ELISA. In addition, the protein expression levels of low-density lipoprotein receptor-related protein (LRP) $5, \beta$-catenin and runt-related transcription factor (Runx) 2 , which are involved in the Wnt/ $\beta$-catenin signaling pathway, were detected by western blot analysis. The results revealed that the GV and BL EA treatment groups significantly increased the serum levels of E2 and OC, decreased the serum levels of TRACP $5 b$ and increased the BMD of the lumbar vertebra when compared with the OVX group. With regard to the histomorphology of the bone tissue, an ordered arrangement and a slight thinning of the trabeculae, with no evident hairline fractures, was observed in the femurs following EA treatment in the OVX rats. Furthermore, histomorphological analysis revealed that EA treatment significantly increased the values of the bone histomorphometry indices, trabecular area percentage, trabecular thickness and trabecular number, and reduced the value of trabecular separation, as compared with the OVX group. In addition, the ratio of OPG/RANKL expression was significantly upregulated, and the expression levels of LRP5, $\beta$-catenin and Runx 2 were significantly upregulated in the EA treatment groups. Therefore, these results demonstrated that long-term stimulation with EA treatment at the GV and BL acupoints was able to alleviate osteoporosis induced by an ovariectomy through regulating the OPG/RANKL and $\mathrm{Wnt} / \beta$-catenin signaling pathways.

\section{Introduction}

Osteoporosis, which is defined as a systemic skeletal impairment characterized by low bone mass, the deterioration of bone tissues and an increased risk of fracture, is a universal major public health problem $(1,2)$. Osteoporosis is affected by numerous factors, including age, genetic background and sex hormones. Postmenopausal osteoporosis (PMOP), a type of osteoporosis that is considered to be associated with an ovarian hormone deficiency, is by far the most common cause 
of age-associated bone loss (3). As estrogen levels decrease, an increase in bone breakdown occurs, which is associated with bone formation, microarchitectural deterioration and decreased bone mass (4). Statistics show that $40 \%$ of females aged $>50$ years suffer from an osteoporotic fracture during their lifetime (5). Estrogen plays a critical role in maintaining bone restoration, particularly for females, and estrogen hormone replacement therapy (HRT) has been proposed as the most effective therapeutic method for the prevention and treatment of PMOP for decades (6). However, prolonged HRT therapy is not well accepted due to the various side effects, which include higher occurrences of breast carcinoma, endometrial cancer and cardiovascular diseases (7). Therefore, the development of a novel, safer and more effective bone protective treatment remains a focus of experimental and clinical research.

Electroacupuncture (EA) is a modified acupuncture method that is a specified Chinese medical therapy based on the principles of regulating Yin and Yang and removing Qi stagnation and blood stasis of the channels and collaterals. In China, EA is considered to be an effective, alternative and complementary therapy for the treatment of a multitude of different human diseases (8). Clinical trials have indicated that acupuncture is an effective treatment for menopausal and perimenopausal syndromes, as well as osteoporosis (9-11). In addition, experimental studies have shown that pretreatment with EA not only increases the bone mineral density (BMD), but can also prevent bone loss in ovariectomized (OVX) rats $(12,13)$. In addition, EA pretreatment has been demonstrated to promote bone healing and callus formation in a rat model of tibia fracture (14). However, whether EA treament at the acupoints of governor vessel $(\mathrm{GV})$ and bladder meridian (BL) is able to ameliorate the process of PMOP in OVX rats is yet to be determined, and if so, the underlying mechanisms require investigation.

Previously, the osteoprotegerin (OPG)/receptor activator of nuclear factor $\kappa \mathrm{B}$ ligand (RANKL) signaling pathway was found to be involved in the regulation of osteoblastogenesis and osteoblast activity, which was regarded as a key factor in inhibiting bone proliferation. Furthermore, the signaling pathway was found to directly participate in the process of bone formation and bone resorption $(15,16)$. Homeostatic bone remodeling requires the coordinated integration of biological signals from numerous cellular signaling transduction pathways, and these pathways may be exploited in the development of novel therapies for osteoporosis (17). The canonical Wnt/ $\beta$-catenin signaling pathway, which controls multiple developmental processes in joint and skeletal patterning, may also be involved in the progression of osteoporosis $(18,19)$.

In accordance with the aforementioned observations and studies, the aim of the present study was to investigate the effects of EA at the acupoints of GV and BL through analyzing the serum levels of estradiol (E2), the bone turnover markers, osteocalcin (OC) and tartrate-resistant acid phosphatase 5b (TRACP 5b), as well as the BMD and bone microarchitecture. In addition, the expression levels of certain important components of the OPG/RANKL and Wnt/ $\beta$-catenin signaling pathways were investigated, with the aim of providing a theoretical understanding for the clinical treatment of PMOP.

\section{Materials and methods}

Reagents. Antibodies targeting low-density lipoprotein receptor-related protein (LRP) 5 (cat. no. sc-390267), $\beta$-catenin (cat. no. sc-79637), runt-related transcription factor (Runx) 2 (cat. no. sc-10758) and $\beta$-actin (cat. no. sc-47778) were obtained from Santa Cruz Biotechnology, Inc. (Santa Cruz, CA, USA). Clarity $^{\mathrm{TM}}$ western enhanced chemiluminescence substrate (cat. no. 170-5060) was obtained from Bio-Rad Laboratories, Inc., Hercules, CA, USA.

Animals. In total, 40 healthy and clean, female Sprague Dawley rats (weight, $326 \pm 14 \mathrm{~g}$; age, 6 months) were purchased from the Shanghai Laboratory Animal Center [Shanghai, China; Laboratory Animal Use Certificate no. SCXK (SH) 2007-0005]. The rats were housed in a sterile environment. Experiments involving the animals complied with Guidance Suggestions for the Care and Use of Laboratory Animals (2006), administered by the Ministry of Science and Technology in China (20).

Animal models and experimental groups. After a week of adaptability feeding, 10 rats were randomly assigned to the sham-operated (sham) group. The remaining 30 rats were assigned to the OVX group. The OVX rats were anesthetized with $2 \%$ pentobarbital sodium (cat. no. P3761; Sigma-Aldrich, St. Louis, MO, USA), after which both ovaries were removed. The sham rats underwent surgery in which the fat tissue near the ovaries was resected. Following surgery, 50,000 units of penicillin were administered intramuscularly each day for three days. Three months after the surgery (when the formation of osteoporosis was expected), the OVX rats were randomly divided into three groups of 10 rats each. The ovariectomized (OVX) control group did not receive any additional treatment. Two EA treatment groups were established, one group assessing the Mingmen and Jizhong acupoints (GV group), and the other group assessing the Pishu and Shenshu acupoints (BL group). The experimental procedures were in compliance with the Animal Protection Law of China (2001), and the study was approved by the Ethics Committee at the Fujian University of Traditional Chinese Medicine (Fuzhou, China).

EA treatment. EA procedures were performed on lightly anesthetized rats (with $2 \%$ pentobarbital sodium), as described previously, to minimize the stress induced by the animal restraint that was necessary for needle insertion and stimulation. The selected acupoints in the GV group were Mingmen (GV4) and Jizhong (GV6), while in the BL group, Pishu (BL20) and Shenshu (BL23) were selected. Disposable stainless steel acupuncture needles $(0.24 \times 30 \mathrm{~mm}$; Suzhou Huatuo Medical Equipment Co., Ltd., Suzhou, China) were inserted perpendicularly into the acupuncture points at a depth of 5-10 $\mathrm{mm}$. The stimuli were generated by an EA apparatus (Huatuo SDZ-V type; Suzhou Huatuo Medical Equipment Co., Ltd.) with $2 / 100 \mathrm{~Hz}$ alternating frequency. The EA treatment was administered once daily for 20 min each time, with 10 treatments constituting a therapeutic course. Between each 10-day course, there was an interval of 5 days. The treatment period lasted for 90 days. The sham group rats received no treatment. 

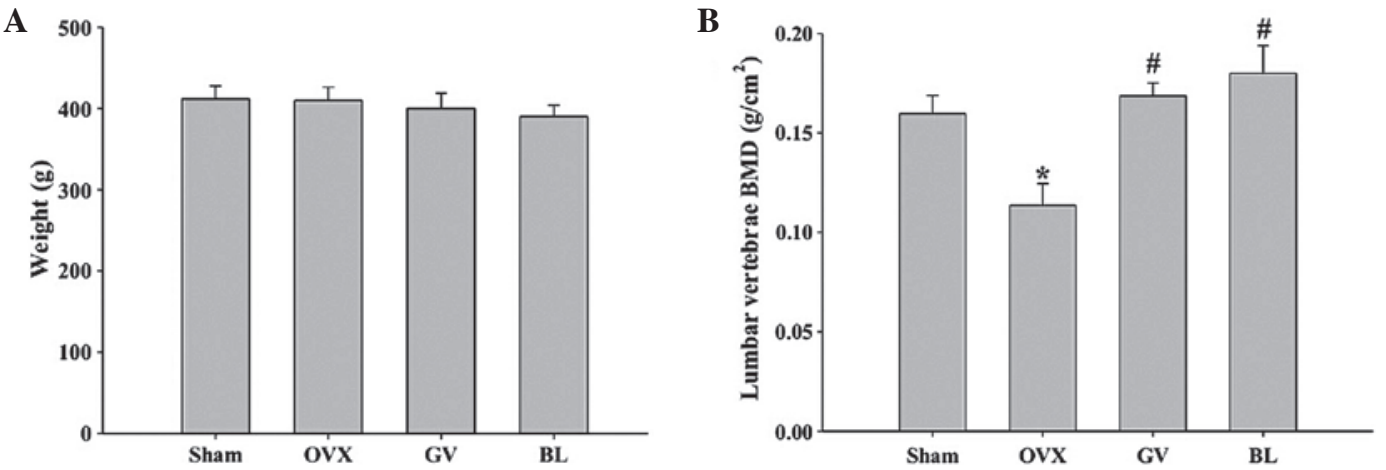

Figure 1. Effect of electroacupuncture on bone loss in OVX rats. (A) Body weights of the rats recorded at the end of the experimental period. (B) Bone mineral density of the lumbar vertebrae. Data are presented as the mean \pm standard deviation $(\mathrm{n}=10)$. ${ }^{*} \mathrm{P}<0.01$, vs. sham group; ${ }^{*} \mathrm{P}<0.01$, vs. OVX group. OVX, ovariectomized; GV, governor vessel; BL, bladder meridian; BMD, bone mineral density.

Bone densitometry analysis. At the end of the study, rats were deeply anesthetized with $10 \%$ chloral hydrate solution $(0.3 \mathrm{ml} / 100 \mathrm{~g}$, i.p.). Following sacrifice of the rats, the lumbar vertebrae (L5) were removed. BMD was determined by dual-energy X-ray absorptiometry (Lunar-DPX-IQ; GE Medical Systems, Madison, WI, USA), which was equipped with small animal measurement software for bone density assessment.

Bone histomorphology analysis. Histomorphology analysis was performed to observe the changes in the bone tissue. The left femurs were removed and fixed with a $10 \%$ ethylenediaminetetraacetic acid solution ( $\mathrm{pH} 7.4$ ) at $4^{\circ} \mathrm{C}$ for 3 weeks. Following decalcification, each bone sample was cut into $3-\mu \mathrm{m}$ coronal planes and embedded in paraffin for tissue sectioning. The sections were stained with hematoxylin and eosin (H\&E) to evaluate the changes in the bone. A histomorphometric study of the femurs was performed using image analysis software (Image-Pro Plus 6.1 for Windows; Media Cybernetics, Inc., Rockville, MD, USA). The parameters measured included the mean thickness of the trabeculae (Tb.Th, $\mu \mathrm{m}$ ), the trabecular area (Tb.Ar, \%), the trabecular bone number (Tb.N, $\left.\mathrm{mm}^{-1}\right)$ and the trabecular separation (Tb.Sp, mm).

Assessment analysis of the serum levels of E2 and the bone turnover markers, OC and TRACP 5b. Following the 90-day EA treatment period, blood samples were collected from all the experimental animals and centrifuged at 3,000 x g for $20 \mathrm{~min}$ at $4^{\circ} \mathrm{C}$. The supernatants were stored at $-20^{\circ} \mathrm{C}$ until required for analysis. Subsequently, the serum E2, OC and TRACP 5b levels were observed using an ELISA kit (Wuhan Elabscience Biotechnology Co., Ltd., Wuhan, China), according to the manufacturer's instructions.

Serum $O P G$ and RANKL expression levels. Serum levels of OPG and RANKL were assessed using an ELISA (Quantikine; R\&D Systems, Inc., Minneapolis, MN, USA), according to the manufacturer's instructions. The ratio of OPG to RANKL, expressed as OPG/RANKL, was used as a measure to describe the process of bone formation coupled with bone resorption.

Western blot analysis for the determination of LRP5, $\beta$-catenin and Runx 2 protein expression. Protein expression levels of LRP5, $\beta$-catenin and Runx 2 in the femur tissues obtained from the rats in the different groups were detected by western blot analysis. Protein was extracted from the femurs using a lysis buffer that contained a protease inhibitor cocktail (Roche Diagnostics GmbH, Mannheim, Germany). Lysates were centrifuged for $15 \mathrm{~min}$ at $12,000 \mathrm{x} \mathrm{g}$ to obtain the supernatants for further analysis. The protein concentration of the lysates was measured using a bicinchoninic acid quantification assay (Pierce Biotechnology, Inc., Rockford, IL, USA). Proteins (50 $\mu \mathrm{g}$ ) were separated using 10\% SDS-PAGE, and transferred to polyvinylidene fluoride membranes with a 0.45- $\mu \mathrm{m}$ pore size (IPVH00010; EMD Millipore, Billerica, MA, USA). The membranes were incubated with monoclonal primary antibodies targeting LRP5, $\beta$-catenin, Runx 2 and $\beta$-actin $(1: 1,000)$, which were diluted in immunoblot buffer [Tris-buffered saline containing 0.05\% Tween-20 (TBST) and $5 \%$ non-fat dry milk], overnight at $4^{\circ} \mathrm{C}$. Following washing with TBST three times, the membranes were incubated with an anti-mouse (or rabbit) secondary antibody (anti-mouse IgG, cat. no. sc-2005; anti-rabbit IgG, cat. no. sc-2004; Santa Cruz Biotechnology) conjugated to horseradish peroxidase $(1: 1,000)$ for $1 \mathrm{~h}$ at room temperature. Following washing, the blots were visualized with a Clarity ${ }^{\mathrm{TM}}$ western enhanced chemiluminescence substrate (Bio-Rad Laboratories) for 1 min using a camera along with the ChemiDoc XRS+ System (Bio-Rad Laboratories, Inc.). The pixel intensities of the immunoreactive bands were quantified using the percentage-adjusted volume feature of the Image Lab software (Bio-Rad Laboratories, Inc.). $\beta$-actin served as an internal control.

Statistical analysis. All results are represented as the mean \pm standard deviation of $\geq 3$ experiments. Data were analyzed using one-way analysis of variance using SPSS statistical package (version 16.0, SPSS, Inc., Chicago, IL, USA). $\mathrm{P}<0.05$ was considered to indicate a statistically significant difference.

\section{Results}

Body weight and BMD. No statistically significant difference was observed in the body weight among the groups in the initial experimental period. In addition, at the end of the experimental period, the body weights in the OVX 

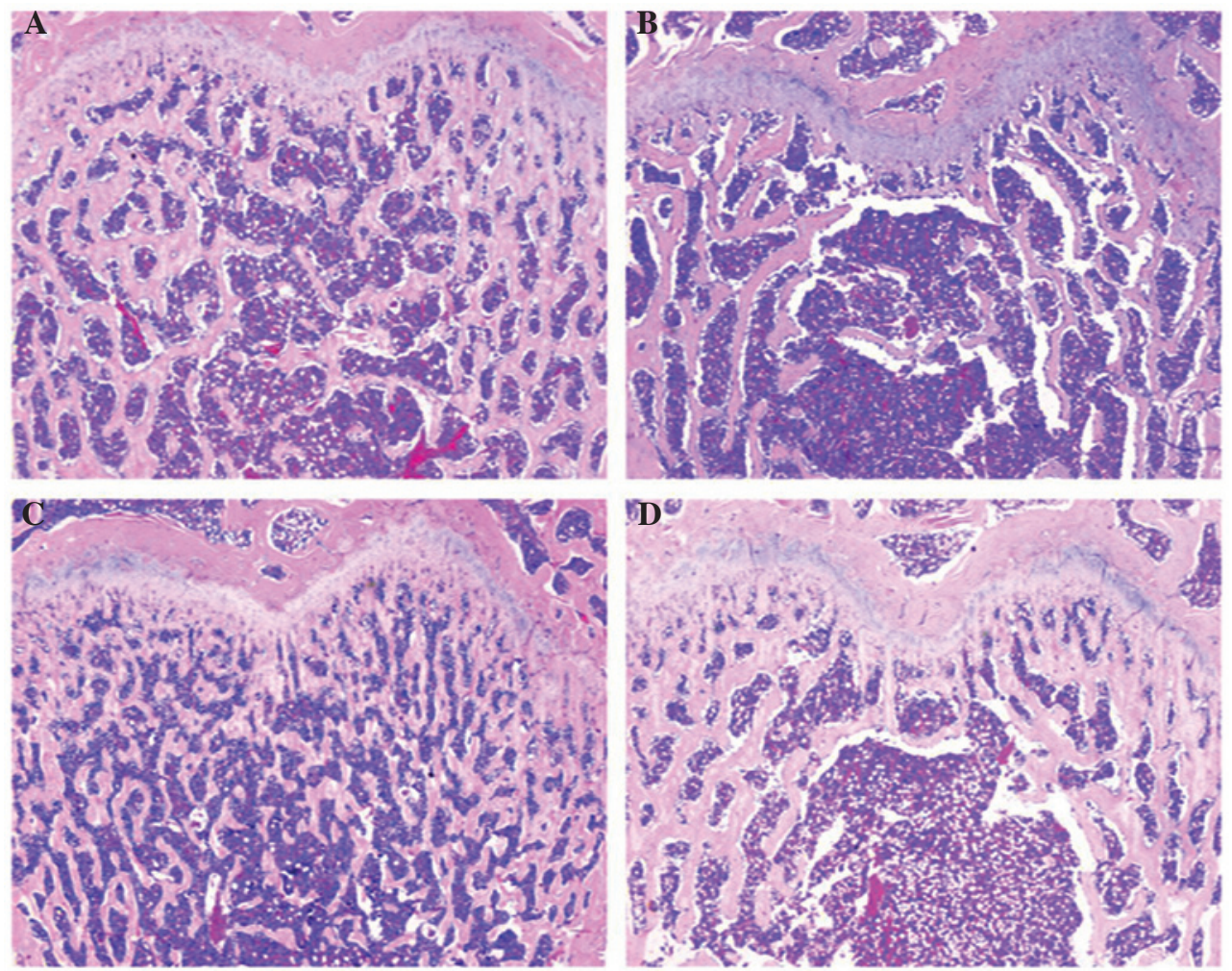

Figure 2. Hematoxylin and eosin (H\&E) staining of the femurs. Following treatment with or without electroacupuncture for 90 days, the rats were sacrificed and specimens of the left femur from each group were processed for H\&E staining. The images are representative (magnification, $\mathrm{x} 40$ ). (A) Rats in the sham control group show a complete and ordered trabecula structure and no hairline fractures. (B) Rats in the ovariectomized group show a reduced number of disordered and thinning trabeculae, empty bone lacunae and hairline fractures. (C) Rats in the governor vessel group show a complete trabecula structure with a slightly ordered arrangement of trabeculae and no clear hairline fractures. (D) Rats in the bladder meridian group show a complete trabecula structure with a slightly ordered arrangement of trabeculae and no evident hairline fractures.

group and EA treatment groups did not differ significantly when compared with the sham group, indicating that no adverse effects were observed as a result of the EA treatment (Fig. 1A).

As shown in Fig. 1B, the ovariectomy procedure caused a significant reduction in the BMD of the lumbar vertebrae in the OVX rats, as compared with that in the sham-operated group $(\mathrm{P}<0.01)$. GV or BL EA treatment was shown to markedly inhibit the OVX-induced decreases in BMD of the lumbar vertebrae; however, there was no statistically significant difference between the GV and BL treatment groups.

Histomorphometry analysis. As shown in Fig. 2A, H\&E staining of the femurs in the sham group revealed complete trabecular structures in an ordered arrangement, numerous trabeculae with reasonable diameters, few empty bone lacunae and no hairline fractures. However, in the OVX group (Fig. 2B), there were fewer trabeculae, which were disordered and thinning. In addition, large areas of empty bone lacunae and hairline fractures were observed. In the $\mathrm{GV}$ and BL treatment groups (Fig. 2C and D), the femurs exhibited a complete trabecular structure, a slightly ordered arrangement of trabeculae, slight thinning of the trabeculae, small amounts of empty bone lacunae and no evident hairline fractures.

Histomorphometric parameters of the trabecular bone mass and bone architecture in the femur sections are shown in Fig. 3. The OVX rats exhibited significant reductions in the Tb.Ar, Tb.Th and Tb.N ( $\mathrm{P}<0.01$ for all) and a significant increase in the Tb.Sp $(\mathrm{P}<0.01)$, when compared with the sham group. However, in the GV and BL treatment groups, significantly increased values of Tb.Ar, Tb.Th and Tb.N $(\mathrm{P}<0.01$ for all), and a significantly reduced value of $\mathrm{Tb} . \mathrm{Sp}(\mathrm{P}<0.01)$, were observed, as compared with the OVX group. No statistically significant difference was observed between the GV and BL treatment groups.

Serum levels of E2, OC and TRACP $5 b$. Serum levels of $\mathrm{E} 2$, and the bone formation markers, OC and TRACP $5 \mathrm{~b}$, were analyzed using ELISA to provide an evaluation of the effects of EA on systemic estrogenic and bone metabolism. As shown in Fig. 4, the serum levels of E2 were significantly decreased in the OVX group, while the levels of OC and TRACP 5b were increased when compared with the sham group. GV and BL EA treatments were shown to significantly increase the serum levels of $\mathrm{E} 2$ and $\mathrm{OC}$, while decreasing the serum levels of TRACP $5 \mathrm{~b}$, when compared with the OVX group $(\mathrm{P}<0.01)$. Interestingly, the EA treatments increased the serum levels of OC to a greater extent than the OVX rats, as compared with the sham group. We hypothesise that the high turnover bone metabolism caused the stress response in the GV and BL rats, which can increase the serum level of OC.

Serum levels of $O P G$ and RANKL. As shown in Fig. 5, the serum OPG level was lower $(\mathrm{P}<0.01)$ in the OVX rats 
A

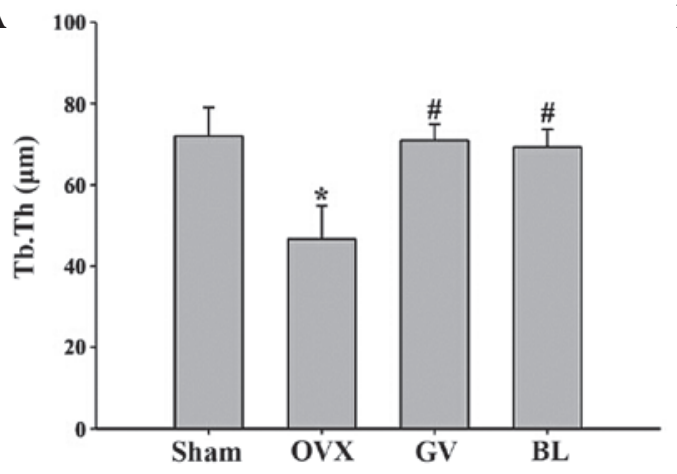

C

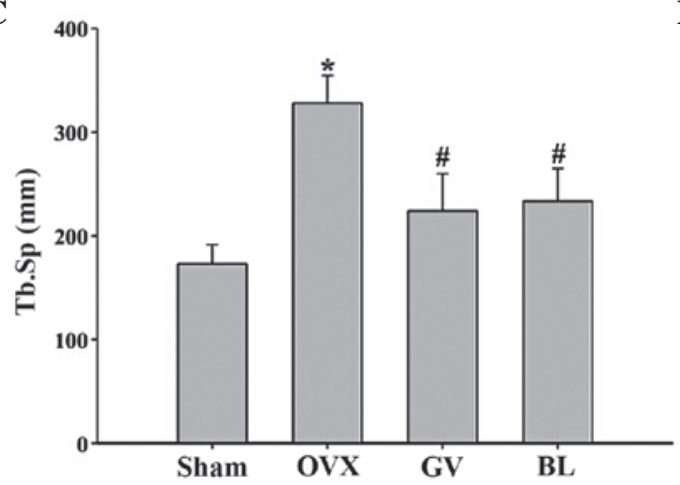

B

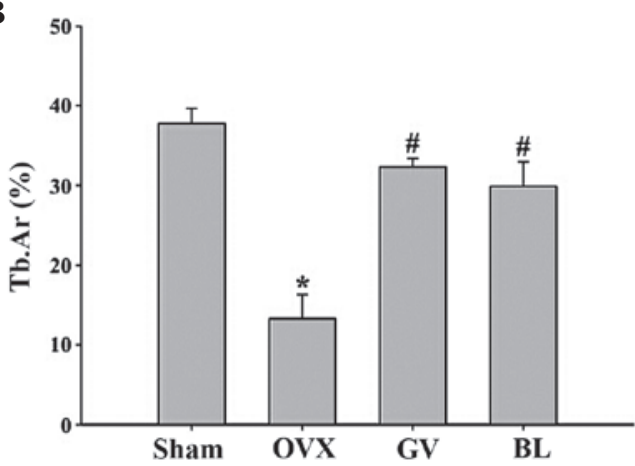

D

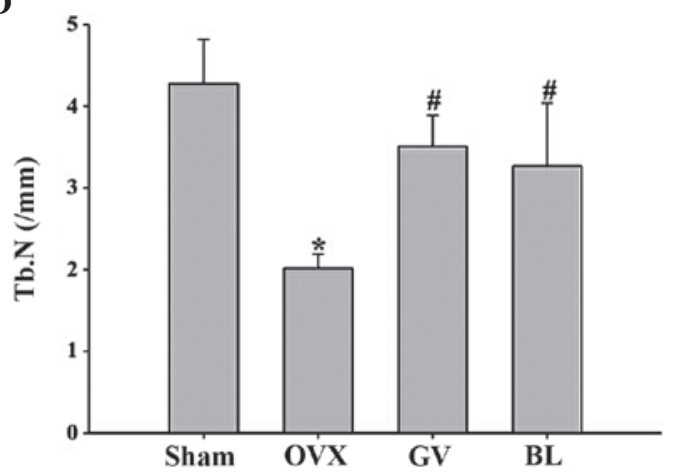

Figure 3. Histomorphometric analysis of the femurs in the various groups. Image analysis software was used to determine the (A) Tb.Th, (B) Tb.Ar percentage; (C) Tb.Sp and (D) Tb.N. Data are presented as the mean \pm standard deviation $(\mathrm{n}=10)$. "P<0.01, vs. sham group; ${ }^{*} \mathrm{P}<0.01$, vs. OVX group. Tb, trabecular; Th, thickness; Ar, area; Sp; separation; N, number; OVX, ovariectomized; GV, governor vessel; BL, bladder meridian.

A

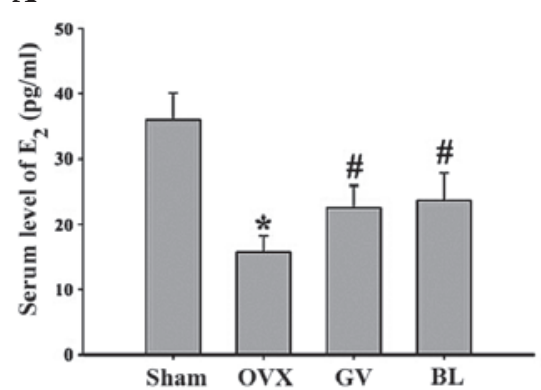

B

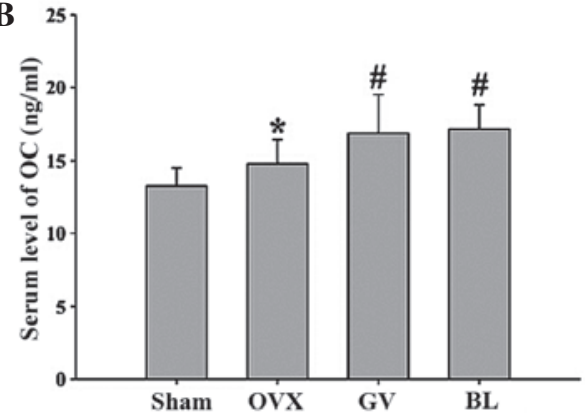

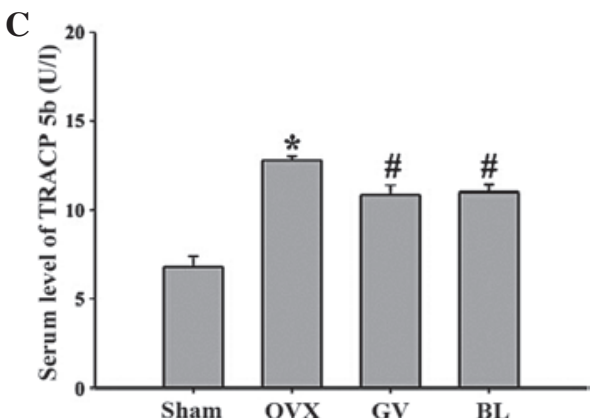

Figure 4. Effects of electroacupuncture on the serum levels of (A) E2 and the bone turnover markers, (B) OC and (C) TRACP 5b, as observed by ELISA. Data are presented as the mean \pm standard deviation $(\mathrm{n}=10)$. " $\mathrm{P}<0.01$, vs. sham group; ${ }^{*} \mathrm{P}<0.01$, vs. OVX group. E2, estradiol; OC, osteocalcin; TRACP, tartrate-resistant acid phosphatase; OVX, ovariectomized; GV, governor vessel; BL, bladder meridian.

$(481.18 \pm 46.08 \mathrm{pg} / \mathrm{ml})$ when compared with the sham-operated animals $(745.0 \pm 54.7 \mathrm{pg} / \mathrm{ml})$. By contrast, estrogen deficiency increased the serum level of RANKL $(\mathrm{P}<0.01)$, while the ratio of OPG/RANKL $(\mathrm{P}<0.01)$ was significantly decreased when compared with the sham group. GV and BL treatment were found to reverse the aforementioned findings. Thus, the ratio of OPG/RANKL was significantly increased following treatment with EA.

Western blot analyses of LRP5, $\beta$-catenin and Runx 2 protein expression. To investigate the role of EA treatment on the Wnt/ $\beta$-catenin signaling pathway and identify the mechanisms involved in the regulation of bone formation and resorption, western blot analyses were performed on bone tissues to examine the protein expression levels of LRP5, $\beta$-catenin and Runx2. The results demonstrated that the femurs from the OVX rats exhibited reduced levels of LRP5, $\beta$-catenin and Runx 2 protein expression when compared with the sham-operated rats. However, following GV and BL treatments, the protein expression levels of LRP5, $\beta$-catenin and Runx2 significantly increased when compared with the OVX group (Fig. 6).

\section{Discussion}

PMOP is associated with a homeostatic imbalance between bone modeling and bone resorption (3). For a number of years, HRT has been used for the treatment and prevention of PMOP; however, this therapeutic method is associated with serious side effects, including higher rates of thromboem- 

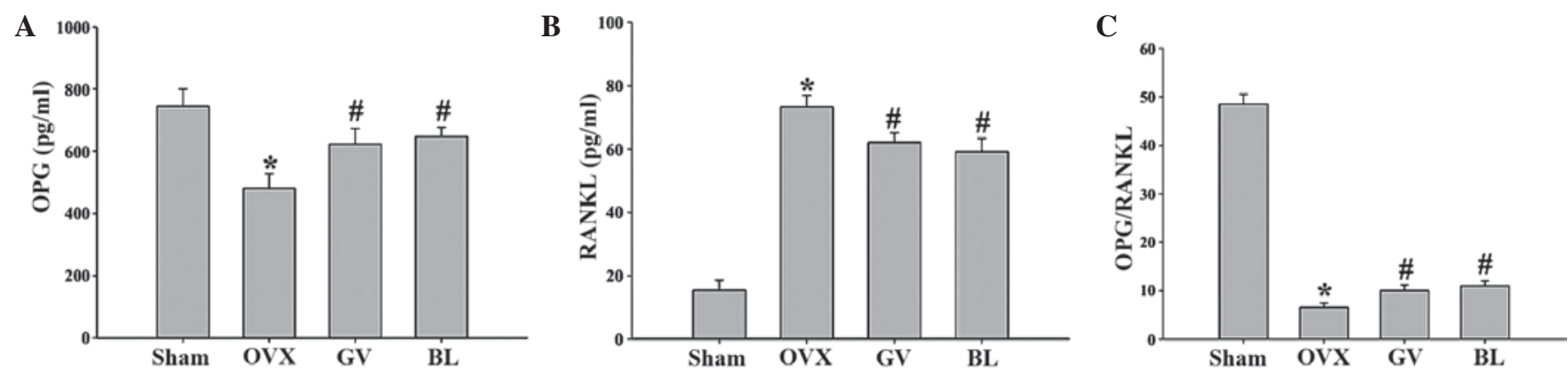

Figure 5. Effect of electroacupuncture on the serum expression levels of (A) OPG and (B) RANKL in the femurs, as assessed by ELISA. (C) Ratio of OPG/RANKL expression in the femurs. Data are presented as the mean \pm standard deviation $(\mathrm{n}=10)$. ${ }^{\text {}} \mathrm{P}<0.01$, vs. sham group; ${ }^{\prime \prime} \mathrm{P}<0.01$, vs. OVX group. OPG osteoprotegerin; RANKL, receptor activator of nuclear factor-кB ligand; OVX, ovariectomized; GV, governor vessel; BL, bladder meridian.

A

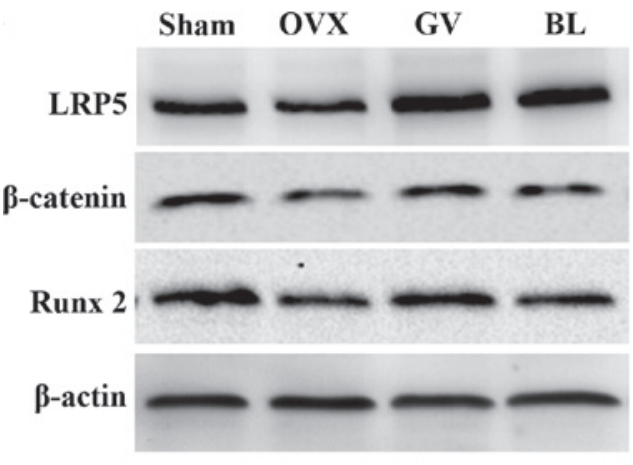

C

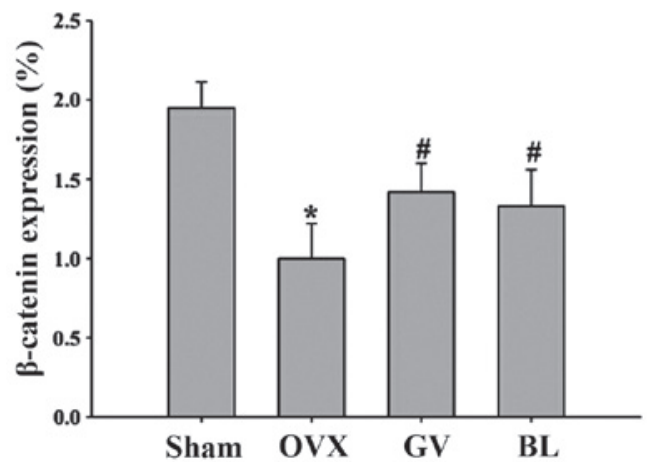

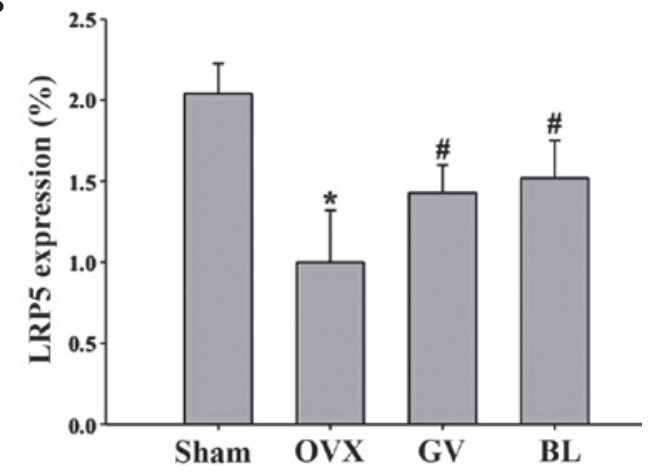

D

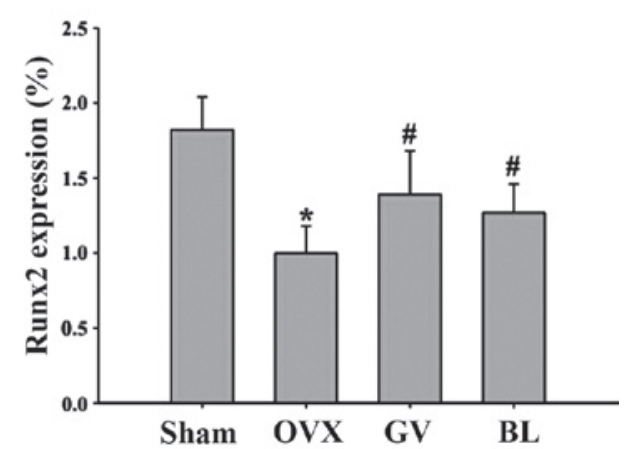

Figure 6. Effects of electroacupuncture on the expression levels of various protein involved in the Wnt/ $\beta$-catenin signaling pathway. (A) Representative western blot, where $\beta$-actin was used as the internal control. Quantitative determination of the protein expression levels of (B) LRP5, (C) $\beta$-catenin and (D) Runx2 in the femurs of the different groups, as determined by western blot analysis. Data are presented as the mean \pm standard deviation. * $\mathrm{P}<0.01$, vs. sham group; ${ }^{\#} \mathrm{P}<0.01$, vs. OVX group. LRP5, low-density lipoprotein receptor-related protein 5; Runx2, runt-related transcription factor 2; OVX, ovariectomized; GV, governor vessel; BL, bladder meridian.

bolism, heart attack, breast and ovarian cancers, stroke and Alzheimer's disease (6). Traditionally, acupuncture has been used in Chinese medicine for the management of disease and pain. Since acupuncture was proposed as a therapeutic intervention of complementary medicine by a National Institutes of Health consensus (21), the efficacy of acupuncture has become more accepted in the western world. EA, which combines the therapeutic effects of traditional manual acupuncture and transcutaneous electric nerve stimulation, can be more easily standardized for frequency, voltage and wave form, in contrast to manual acupuncture (22). Thus, EA has been proposed as a potential alternative to estrogen replacement therapy for the treatment of PMOP due to the decreased incidence of adverse effects.
According to traditional Chinese medicine theory, the 'kidney' controls the bones. A previous study indicated that the incidence of kidney deficiency increases with age, causing a progressive decrease in the BMD (23). In addition, the spleen is considered to be the acquired foundation and source of Qi and blood generation. A spleen deficiency may result in malnourishment of the limbs, other internal organs and bones, with the subsequent result being bone weakness and osteoporosis (24). Therefore, strengthening the spleen and tonifying the kidney have become the theoretical basis for the treatment of PMOP.

In accordance with the traditional Chinese medicine theory, the present study selected Shenshu (BL23) and Mingmen (GV4) to tonify the kidneys, while Pishu (BL20) 
and Jizhong (GV6) were selected to strengthen the spleen. A previous experimental study demonstrated that BL20, BL23 and GV4 were the acupoints most commonly selected for the prevention and treatment of osteoporosis in postmenopausal individuals (25).

The OVX animal model, which exhibits the majority of the characteristics of human PMOP (26), has been widely used as a model for the evaluation of potential osteoporosis treatments (27). The present study was designed to evaluate the effects of EA at the GV and BL acupoints on OVX-induced osteoporosis, and to investigate the effects of EA on the OPG/RANKL/RANK and Wnt//3-catenin signaling pathways. The results of the present study revealed that EA at the GV4 and GV6 or BL20 and BL23 acupoints was able to reduce the loss of bone mass, increase the femoral BMD, regulate the serum levels of the bone turnover markers, OC and TRACP 5b, and improve the microstructure of the bone tissue by increasing the Tb.Ar, Tb.N and Tb.Th and decreasing the Tb.Sp in OVX rats. Furthermore, the EA treatments did not result in any adverse effects with regard to OVX-induced increases in body weight, indicating that the GV and BL treatments exerted beneficial effects on osteoporosis, which may be closely associated with the function of 'strengthening the spleen and tonifying the kidney'.

A number of previous studies (28-30) have implicated the OPG/RANKL/RANK signaling system as a paracrine mediator of mechanical strain on bone metabolism. RANKL, which provides an important signal to osteoclast progenitors, is a membrane-bound molecule of the tumor necrosis factor ligand family that promotes the formation of osteoclasts. The binding of RANKL to its receptor, RANK, which is expressed on osteoclast precursors and mature osteoclasts, induces osteoclastogenesis and the activation of mature osteoclasts. Osteoblasts also synthesize and secrete OPG, a decoy receptor of RANKL, which blocks the interaction between RANKL and RANK, and thereby inhibits osteoclast formation and the activation of mature osteoclasts (28-30). The results of the current study indicated that EA treatment at the GV4 and GV6 or BL20 and BL23 acupoints resulted in the upregulation of OPG expression and the downregulation of RANKL expression and secretion from osteoblasts, subsequently inhibiting osteoclastogenesis and promoting bone formation.

In addition, the Wnt $/ \beta$-catenin signaling pathway plays a key role in the regulation of bone growth and remodeling. When Wnt proteins bind to the Frizzled receptor and the LRP5/6 coreceptor protein, the signaling protein, Dishevelled, becomes activated. Subsequently, the inhibition of glycogen synthase kinase- $3 \beta$ occurs, which inhibits the ubiquitination and degradation of $\beta$-catenin. As a consequence, $\beta$-catenin accumulates in the nucleus and binds to lymphoid enhancer-binding factor- $1 /$ transcription factor to upregulate the expression of Wnt target genes, such as Runx2, which elicits a variety of effects, including the proliferation and differentiation of osteoblasts. In accordance with the results of a previous study (31), the present study observed decreased expression levels of Wnt/ $\beta$-catenin signaling pathway members, including LRP5, $\beta$-catenin, and Runx2, following an ovariectomy. Furthermore, the results revealed that EA treatment at the GV4 and GV6 or BL20 and BL23 acupoints was able to activate the expression of these members. Therefore, Wnt/ $\beta$-catenin signaling has become a focus for the development of targeted therapeutics in PMOP.

In conclusion, to the best of our knowledge, the data presented in the current study demonstrated for the first time the multiple working mechanisms of EA treatment at the acupoints of GV and BL for the therapy of osteoporosis. EA treatment was shown to function through the OPG/RANKL/RANK and Wnt/ $\beta$-catenin signaling pathways, which may be one of the mechanisms through which EA plays an important role in the treatment of PMOP. Therefore, EA was demonstrated to be a potential option in the therapeutic strategy of PMOP, since good tolerance and few undesirable side effects were observed.

\section{Acknowledgements}

The study was supported by a grant from the National Natural Science Foundation of China (no. 81173282).

\section{References}

1. NIH Consensus Development Panel on Osteoporosis Prevention, Diagnosis, and Therapy: Osteoporosis prevention, diagnosis, and therapy. JAMA 285: 785-795, 2001.

2. Kanis JA: Diagnosis of osteoporosis and assessment of fracture risk. Lancet 359: 1929-1936, 2002.

3. Meczekalski B and Czyzyk A: Selective estrogen receptor modulators in treatment of postmenopausal osteoporosis. Ginekol Pol 80: 213-217, 2009 (In Polish).

4. Cole Z, Dennison E and Cooper C: Update on the treatment of post-menopausal osteoporosis. Br Med Bull 86: 129-143, 2008.

5. McNamara LM: Perspective on post-menopausal osteoporosis: Establishing an interdisciplinary understanding of the sequence of events from the molecular level to whole bone fractures. J R Soc Interface 7: 353-372, 2010.

6. Nelson HD: Menopause. Lancet 371: 760-770, 2008.

7. Rossouw JE, Anderson GL, Prentice RL, et al; Writing Group for the Women's Health Initiative Investigators: Risks and benefits of estrogen plus progestin in healthy postmenopausal women: Principal results from The Women's Health Initiative randomized controlled trial. JAMA 288: 321-333, 2002.

8. Han JS: Acupuncture: Neuropeptide release produced by electrical stimulation of different frequencies. Trends Neurosci 26: 17-22, 2003.

9. Mason S, Tovey P and Long AF: Evaluating complementary medicine: Methodological challenges of randomised controlled trials. BMJ 325: 832-834, 2002.

10. Sunay D, Ozdiken M, Arslan H, Seven A and Aral Y: The effect of acupuncture on postmenopausal symptoms and reproductive hormones: A sham controlled clinical trial. Acupunct Med 29: 27-31, 2011.

11. Zhou ZH, Wang NQ, Pan FF, Wu ZH and Dai XY: Clinical observation on combined acupuncture and medication for osteoporosis in postmenopausal women. J Acupunct Tuina Sci 9: 370-375, 2011 (In Chinese).

12. He J, Yang L, Qing Y and He C: Effects of electroacupuncture on bone mineral density, oestradiol level and osteoprotegerin ligand expression in ovariectomised rabbits. Acupunct Med 32: 37-42, 2014.

13. Feng Y, Lin H, Zhang Y, Li L, Wu X, Wang T, Liu Y and Tan Y: Electroacupuncture promotes insulin-like growth factors system in ovariectomized osteoporosis rats. Am J Chin Med 36: 889-897, 2008.

14. Wei YF, Liu YL, Zhang SH, Wang ZO, et al: Effect of electroacupuncture on plasma estrin and bone mineral density in ovariectomized rats. Zhen Ci Yan Jiu 32: 38-41, 2007 (In Chinese).

15. Kong YY, Yoshida H, Sarosi I, et al: OPGL is a key regulator of osteoclastogenesis, lymphocyte development and lymph-node organogenesis. Nature 397: 315-323, 1999.

16. Hofbauer LC and Schoppet M: Clinical implications of the osteoprotegerin/RANKL/RANK system for bone and vascular diseases. JAMA 292: 490-495, 2004. 
17. Hartmann C: A Wnt canon orchestrating osteoblastogenesis. Trends Cell Biol 16: 151-158, 2006.

18. Kim W, Kim M and Jho EH: Wnt/ $\beta$-catenin signalling: From plasma membrane to nucleus. Biochem J 450: 9-21, 2013.

19. Bodine PV and Komm BS: Wnt signaling and osteoblastogenesis. Rev Endocr Metab Disord 7: 33-39, 2006.

20. The Ministry of Science and Technology of the People's Republic of China: Guidance Suggestions for the Care and Use of Laboratory Animals, 2006.

21. Vincent CA and Richardson PH: The evaluation of therapeutic acupuncture: Concepts and methods. Pain 24: 1-13, 1986.

22. Lin JG and Chen WL: Acupuncture analgesia: A review of its mechanisms of actions. Am J Chin Med 36: 635-645, 2008.

23. Zhao Y, Liu KJ, Li JH, Cai XJ and Yang JH: Bone mineral content and kidney asthenia. Chinese J Osteoporosis 2: 19-21, 1996 (In Chinese)

24. Xie L, Yao GH and Guo ZQ: Preliminary study on treatment of osteoporosis with spleen-strengthening and stomach-nourishing method. Hunan Zhongyiyao Xueyuan Xuebao 41: 7-9, 1996.

25. Xu M, Liu BX and Huang CG: The progress of acupuncture treatment on postmenopausal osteoporosis. Zhejiang Zhongyiyao Daxue Xuebao 2: 301-302, 2011.

26. Frolik CA, Bryant HU,Black EC, Magee DE and ChandrasekharS: Time-dependent changes in biochemical bone markers and serum cholesterol in ovariectomized rats: Effects of raloxifene $\mathrm{HCl}$ tamoxifen, estrogen, and alendronate. Bone 18: 621-627, 1996.
27. Sato M, Bryant HU, Iversen $\mathrm{P}$, et al: Advantages of raloxifene over alendronate or estrogen on nonreproductive and reproductive tissues in the long-term dosing of ovariectomized rats. J Pharmacol Exp Ther 279: 298-305, 1996.

28. Yasuda H, Shima N, Nakagawa N, et al: Identity of osteoclastogenesis inhibitory factor (OCIF) and osteoprotegerin (OPG): A mechanism by which OPG/OCIF inhibits osteoclastogenesis in vitro. Endocrinology 139: 1329-1337, 1998.

29. Bord S, Ireland DC, Beavan SR and Compston JE: The effects of estrogen on osteoprotegerin, RANKL, and estrogen receptor expression in human osteoblasts. Bone 32: 136-141, 2003.

30. Lacey DL, Timms E, Tan HL, et al: Osteoprotegerin ligand is a cytokine that regulates osteoclast differentiation and activation. Cell 93: 165-176, 1998.

31. Zhou J, He H, Yang L, Chen S, Guo H, Xia L, Liu H, Qin Y, Liu C, Wei X, Zhou Y and He C: Effects of pulsed electromagnetic fields on bone mass and $\mathrm{Wnt} / \beta$-catenin signaling pathway in ovariectomized rats. Arch Med Res 43: 274-282, 2012. 